\title{
The Design and Analysis of Hexapodal Ant-Bionic Robots
}

\author{
JING Q ${ }^{1}$, CHEN Ya-Ning ${ }^{1}$, SONG Zhong-Kang $^{1}$ and CUI Yu-Lian ${ }^{1}$ \\ ${ }^{1}$ Department of mechanical engineering, The Academy of Armored Forces Engineering, Beijing 100072 , China
}

\begin{abstract}
Herein, we utilize ants as the prototype to build the multi-functional model that can walk, turn, cross obstacles and collect samples under the inventor environment. The operating principle of the robot was introduced and the strength of important elements were checked and discussed to investigate the structure design and the gait analysis of the hexapodal robot. Finally, we realized the motion simulation of the robot in the computer environment.
\end{abstract}

The hexapodal robots have better adaptability to ground and flexibility of walking,suits the complex terrain especially in the world.There is a important significance for military reconnaisance and transport.The article proposes a hexapodal robot design by simulating ants. The design has simple and reliable structure,easy to control,strong practicability.

\section{The structural design of ant robots}

\subsection{The general structure design}

The mechanical instructure of ant-bionic robots is consist of three parts including trunk,leg and belly,as shown in the figure 1.Subject is based on the axis trunk,six legs are located on both sides of the trunk.Belly located on the back of trunk is used to put sample material.The functional instructure includes radar,camera and drill devices,the back of trunk has the radar to detect,the head has a micro-camera,the foot is a high-strength drill ${ }^{[1]}$.

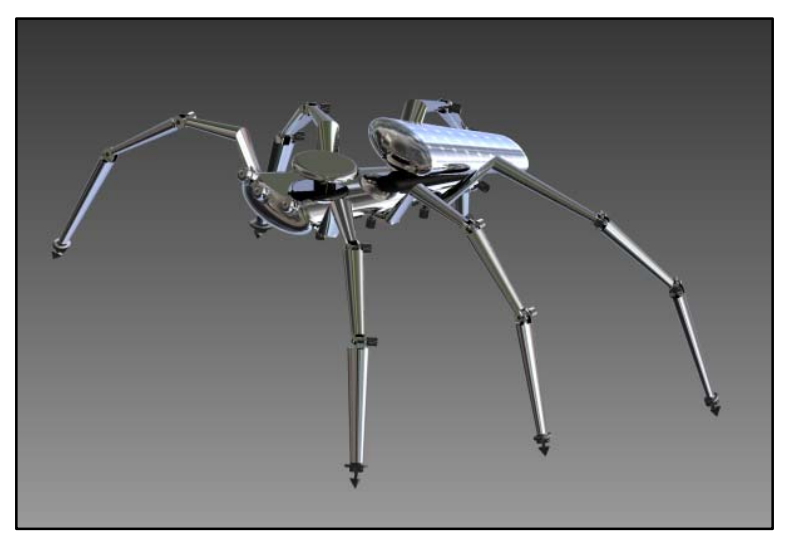

Figure 1. The general structural design of ant robots

\subsection{The leg structure design}

According to the theory of bionic,the structure of leg is consist of hip joint,thigh,middle leg,shank and foot,as shown in the figure 2.Every leg has four degrees of freedom,can complete the motion of raising the body and the directional swing of front and back,high and low,inside and outside.Meanwhile,the drill of foot can rotate with high-speed ${ }^{[2]}$.The connection of rack and pinion is between shank and foot,rack and pinion are used to raise its body when ants cross the obstacle.The motor between foot and shank is used to drive the drill of foot to rotate.

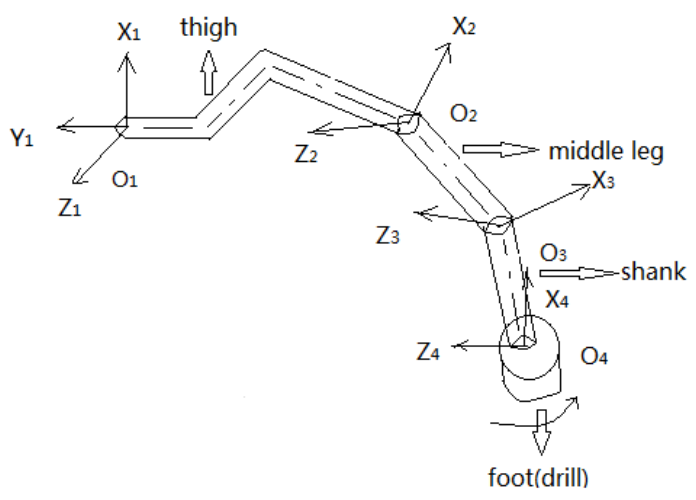

Figure 2. The leg structure design

\subsection{The trunk and belly instructure design}

The trunk is consist of two parts including up and down,installed the radar which use to receive and send signals on the top of trunk.There is the mechanical arm used to sample on the bottom of it.The mechanical arm is made up of three parts to all-around sampling,as shown in the figure 3.We make a sampling place box by simulating the belly of ants at the back of the trunk ${ }^{[3]}$.It can turn down to place the sample which the mechanical arm sample,such as stone,etc.Its surface covers solar panel and produces the energy to meet their activities by solar panel $^{[4]}$.

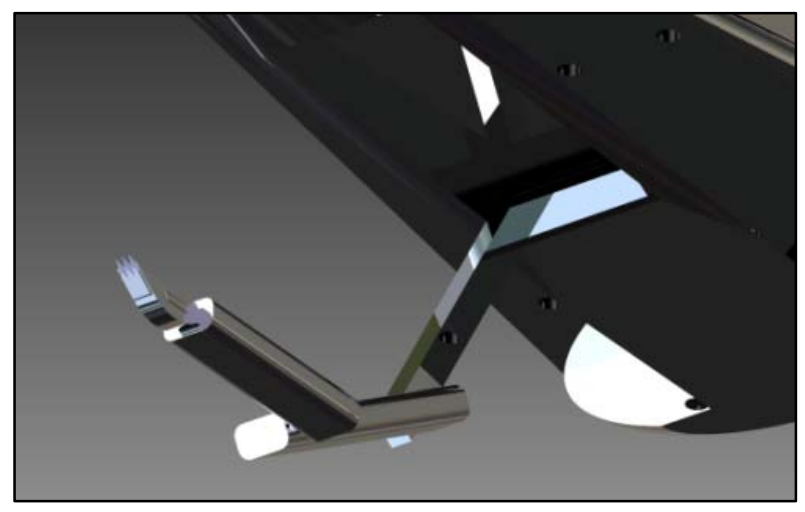

Figure 3. The mechanical belly arm 
According to the different strength and stiffness demand using different material for the different part:the trunk of robot uses alloy steal;the thigh uses high strength and low alloy to make,it can satisfy the driving requirement of hip joint motor;the middle leg and shank use alloy steal.

\section{The finite element analysis}

\subsection{The thigh stress analysis results}

The thigh bear force and moment is mainly motor torque of the hip to hip pressure, body weight and the ground through the leg support at the thigh, through to the ant its weight calculation and estimate, run stress analysis to get the following results, as shown in figure 4 .

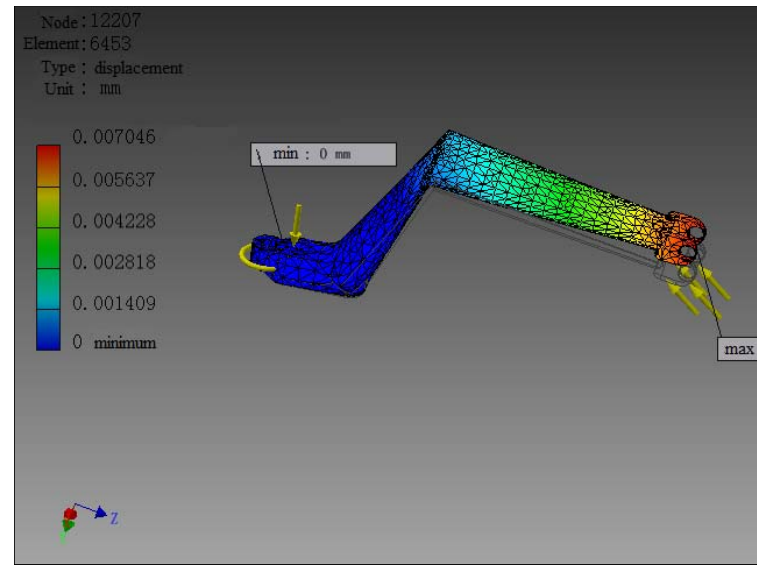

Figure 4. Displacement diagram

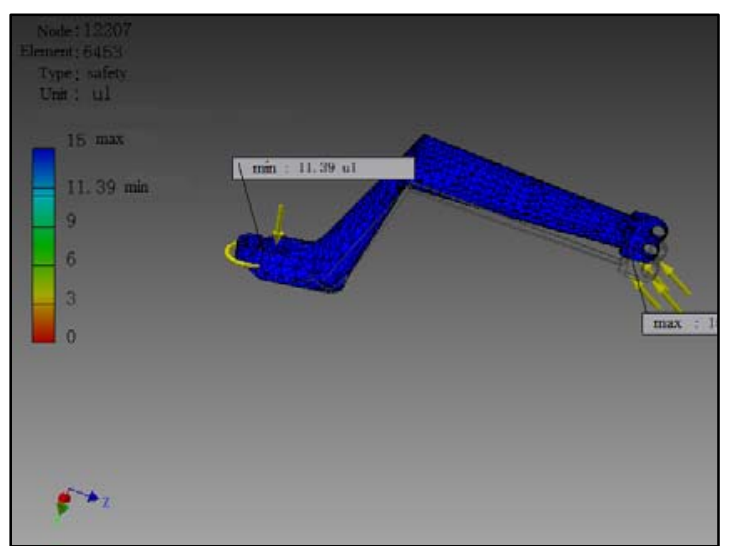

Figure 5. Safety factor

Through the stress analysis, we can learn that the maximum equivalent stress is $24.21 \mathrm{MPa}$, the minimum value of $0.01 \mathrm{MPa}$; The maximum displacement is $0.007046 \mathrm{~mm}$, can be neglected;A maximum of $15 \mathrm{ul}$, minimum safety factor of 11.39 ul.You can see the thigh conform to the requirements of the structure design of ants and carry some weight.

\subsection{The drill stress analysis results}

Drill's main purpose is to compare grinding with hard ground or sample, then sampling, mainly bear is torque and the legs and the pressure of the body, the results of the analysis as shown in figure 6:

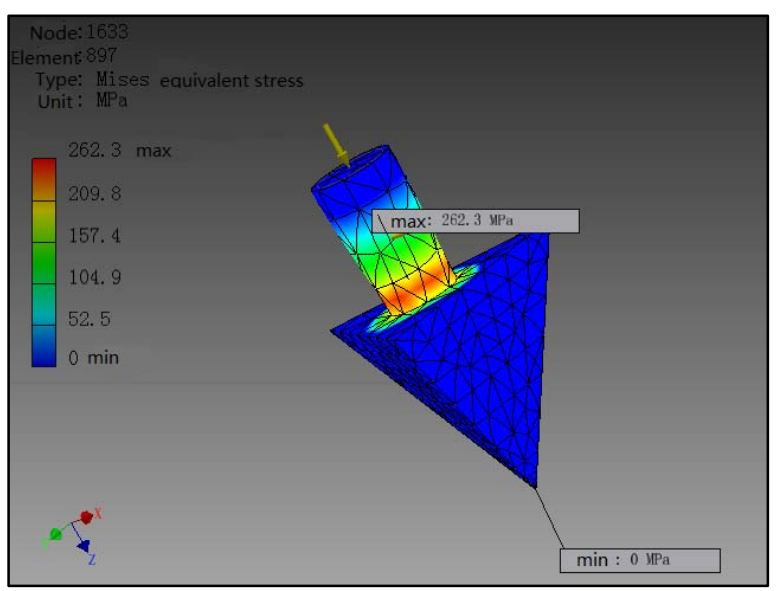

Figure 6. Equivalent stress

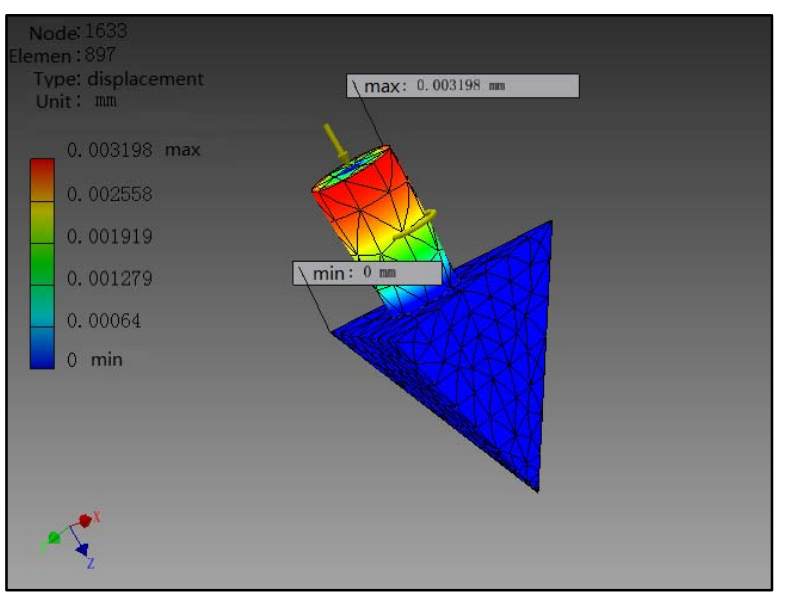

Figure 7. Displacement diagram

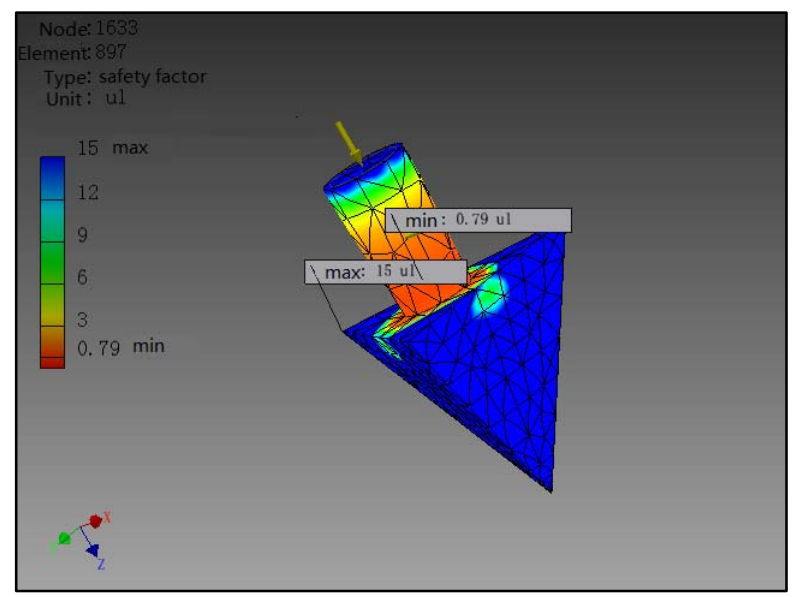

Figure 8. Safety factor

Through the stress analysis, we can learn that the maximum equivalent stress and the minimum is 262.3 $\mathrm{MPa}$, respectively $0 \mathrm{MPa}$.; The maximum displacement is $0.003198 \mathrm{~mm}$, the minimum value of 0 ; Safety factor of the maximum and the minimum $15 \mathrm{ul}$, respectively, 0.79 
ul.Can see bit conform to the requirements of the structure design of ants, able to bear the driving torque of motor, and crushed stones, etc.

\section{Motion simulation and analysis}

Use Inventor to build 3D model,enter the inventor/ simulation module,add motion constraints and drive,start the motion simulation of the robot moving.

The thigh and middle legs' driving use the angle position relationship,the curve of thigh motion simulation is shown in the figure 9, the curve track of middle leg straight walking and obstacle crossing is shown in the figure 10 and 11.We can know from figure 9,10,11,ant robots'thighs swing the angle of 40 degrees when they walk straight and swing front and back at a constant speed.The middle leg swings from side to side at a constant speed when it walks straight; the range of swing has a increase when the robots cross the obstacle.

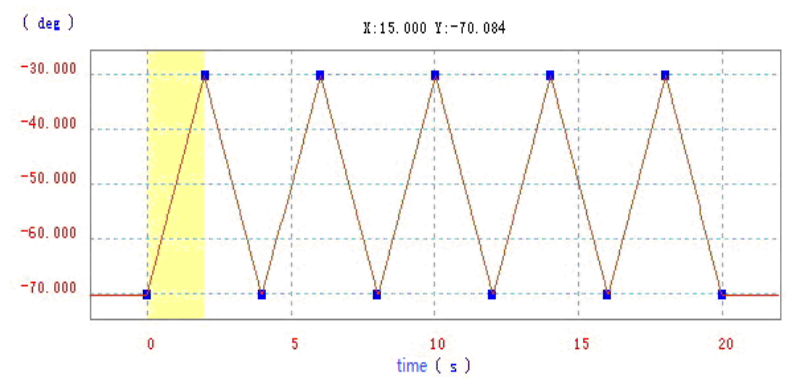

Figure 9. The curve of thighs walking

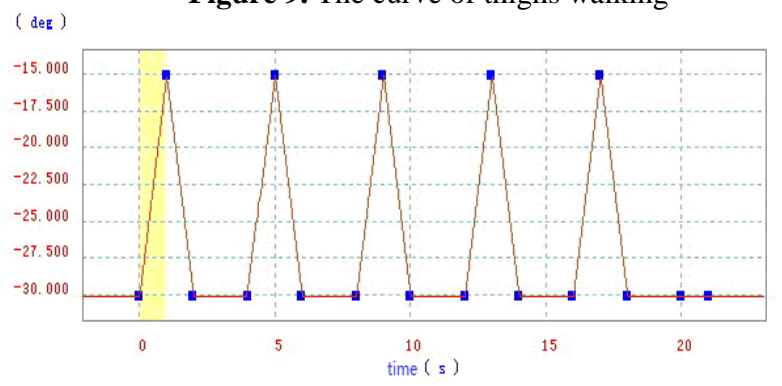

Figure 10. The curve of middle legs walking

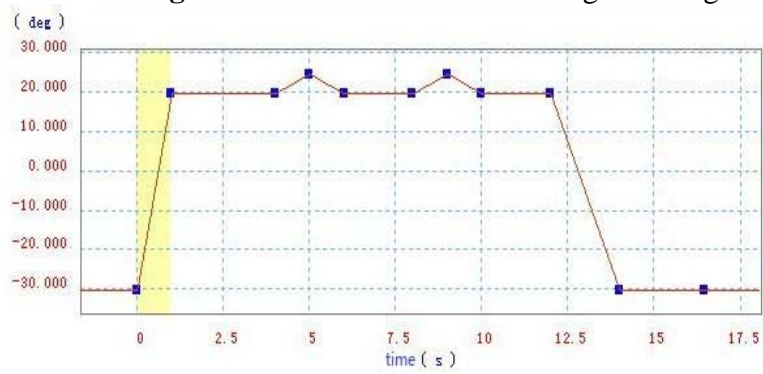

Figure 11. The curve of middle legs obstacle

Leg movement curve of each part through the above analysis, the ants are met in each state (including walk straight, surmounting obstacles, turning) at the time of the force and movement request, can ensure the normal state of ants bionic robot.

\section{Conclusion}

We propose a design scheme of hexapodal robots on the basis of simulating ants.The design has simple instructure,stable connection,strong practicability.The result of simulating shows robots can complete the relevant walking motion according to the require of control system and achieve the motions of straight walking,shinning and obstacle crossing.Captions should be typed in 9-point Times. They should be centred above the tables and flush left beneath the figures.

\section{References}

[1] Xu Xiaoyun,Yan Guozheng,Liu Hua.The research of hexapodal ant-bionic robots[J].2002,9.

[2] Yin Jiying,He Guangping.Articulated Robot[M],Bei jing:Chemical Industry Press,2003.

[3] Zeng Guiying,Liu Shaojun.The design and research of hexapodal walking robot,Machine tools and hydraulic[J],2006,6.

[4] Xu Kai.Humanoid robot gait planning algorithm and implementation[D].Beijing:Tsinghua university,2004. 